\title{
Design of the large hadron electron collider interaction region
}

\author{
E. Cruz-Alaniz ${ }^{*}$ and D. Newton \\ University of Liverpool, Liverpool L69 3BX, United Kingdom \\ and Cockcroft Institute, Daresbury Laboratory, Warrington WA4 4AD, United Kingdom \\ R. Tomás \\ CERN, CH 1211 Geneva 23, Switzerland \\ M. Korostelev \\ Department of Physics, Lancaster University, Lancaster LA1 4YB, United Kingdom \\ and Cockcroft Institute, Daresbury Laboratory, Warrington WA4 4AD, United Kingdom
}

(Received 3 June 2015; published 5 November 2015)

\begin{abstract}
The large hadron electron collider ( $\mathrm{LHeC}$ ) is a proposed upgrade of the Large Hadron Collider (LHC) within the high luminosity LHC (HL-LHC) project, to provide electron-nucleon collisions and explore a new regime of energy and luminosity for deep inelastic scattering. The design of an interaction region for any collider is always a challenging task given that the beams are brought into crossing with the smallest beam sizes in a region where there are tight detector constraints. In this case integrating the LHeC into the existing HL-LHC lattice, to allow simultaneous proton-proton and electron-proton collisions, increases the difficulty of the task. A nominal design was presented in the the LHeC conceptual design report in 2012 featuring an optical configuration that focuses one of the proton beams of the LHC to $\beta^{*}=10 \mathrm{~cm}$ in the LHeC interaction point to reach the desired luminosity of $L=10^{33} \mathrm{~cm}^{-2} \mathrm{~s}^{-1}$. This value is achieved with the aid of a new inner triplet of quadrupoles at a distance $L^{*}=10 \mathrm{~m}$ from the interaction point. However the chromatic beta beating was found intolerable regarding machine protection issues. An advanced chromatic correction scheme was required. This paper explores the feasibility of the extension of a novel optical technique called the achromatic telescopic squeezing scheme and the flexibility of the interaction region design, in order to find the optimal solution that would produce the highest luminosity while controlling the chromaticity, minimizing the synchrotron radiation power and maintaining the dynamic aperture required for stability.
\end{abstract}

DOI: 10.1103/PhysRevSTAB.18.111001

PACS numbers: 41.85.-p

\section{INTRODUCTION AND MOTIVATION}

The Large Hadron Collider (LHC) [1] at CERN with circumference of $27 \mathrm{~km}$ has been providing proton-proton collisions between $\sqrt{s}=7 \mathrm{TeV}$ and $13 \mathrm{TeV}$, lead-lead collisions at $\sqrt{s}=2.76 \mathrm{TeV} /$ nucleon and proton-lead collisions at $\sqrt{s}=5 \mathrm{TeV} /$ nucleon in four different interaction points, ATLAS [2] in interaction point 1 (IP1), ALICE [3] in interaction point 2 (IP2), CMS [4] in interaction point 5 (IP5) and $\mathrm{LHCb}$ [5] in interaction point 8 (IP8). Each of these interaction points is located in its corresponding interaction region (IR). Other sections of the ring, the interaction region 3 (IR3) and interaction region 7 (IR7) are reserved for collimation purposes. A layout of the LHC configuration is shown in Fig. 1.

*e.cruz-alaniz@liverpool.ac.uk

Published by the American Physical Society under the terms of the Creative Commons Attribution 3.0 License. Further distribution of this work must maintain attribution to the author(s) and the published article's title, journal citation, and DOI.
Particle physics has profited in the past from nucleonnucleon, lepton-nucleon and lepton-lepton interactions. The LHC has brought nucleon-nucleon collisions into the $\mathrm{TeV}$ era and the high luminosity Large Hadron Collider (HL-LHC) [6] project aims to build on this success by increasing the luminosity to $5 \times 10^{34} \mathrm{~cm}^{-2} \mathrm{~s}^{-1}$ in IP1 and IP5.

The large hadron electron collider $(\mathrm{LHeC})$ aims to make use of the LHC infrastructure and a new $60 \mathrm{GeV}$ electron accelerator to provide electron-proton (e-p) collisions, also, in the $\mathrm{TeV}$ scale, aiming for an energy $(\sqrt{s}=2 \mathrm{TeV})$ 4 times higher and a luminosity $\left(10^{33} \mathrm{~cm}^{-2} \mathrm{~s}^{-1}\right) 100$ times higher than the previous e-p collider, HERA [7]. The LHeC would work alongside the HL-LHC and provide a complementary set of measurements.

This paper starts by describing the method to achieve high luminosity collisions in the HL-LHC experiments using the achromatic telescopic squeezing (ATS) scheme in Sec. II. The nominal LHeC IR design status as it was presented in the $\mathrm{LHeC}$ conceptual design report (CDR) is described in Sec. III, at this point the integration into the HL-LHC lattice was not yet successful. This paper 


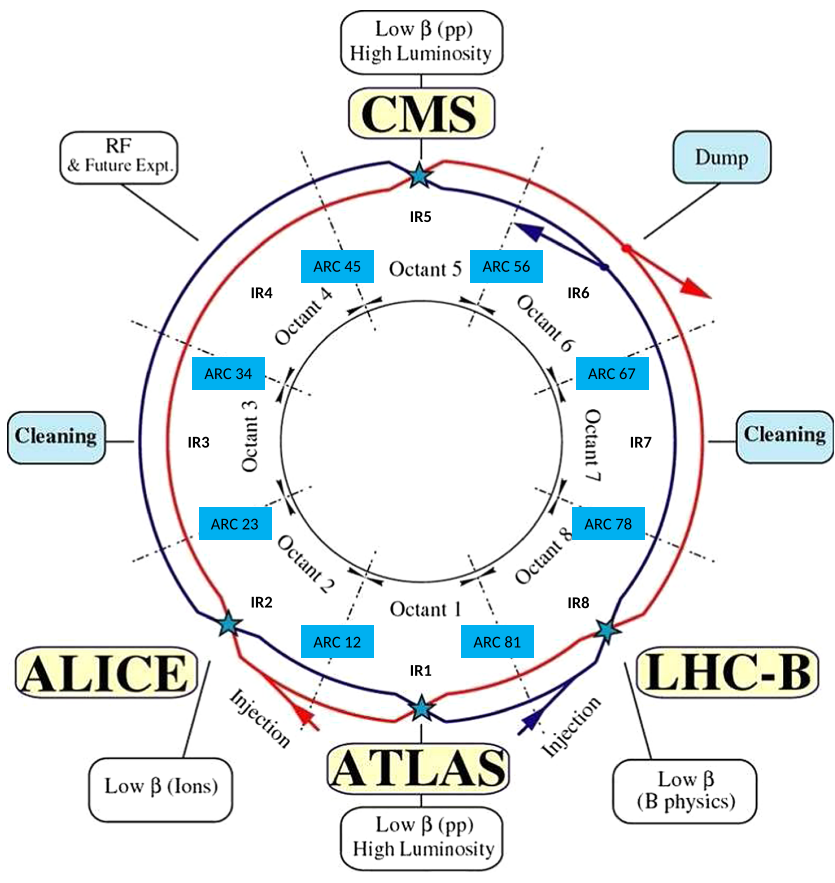

FIG. 1. LHC schematic configuration showing clockwise beam 1 colliding with counterclockwise beam 2 in the four different experiments [1].

demonstrates the feasibility of the extension of the ATS scheme into the LHeC IR, as described in Sec. IV. The flexibility of this design is also studied to explore the limits on luminosity and the reduction of the synchrotron radiation in the IR (Sec. V). Most importantly the chromatic correction of the previous optical designs is addressed and its limits explored (Sec. VI). The synchrotron radiation is studied to avoid too large background in the detector (Sec. VII). Lastly, particle tracking studies are done to study the feasibility of such designs in terms of the stability of the beam (Secs. VIII and IX).

\section{HIGH LUMINOSITY COLLISIONS}

The following sections introduce the HL-LHC current design, to later extend the study with the $\mathrm{LHeC}$ insertion in IR2.

\section{A. Minimizing $\beta^{*}$ in HL-LHC}

The upgrade plans for the HL-LHC project include the implementation of a new inner triplet (IT) of high aperture quadrupoles. This inner triplet comprises three quadrupoles (Q1, Q2 and Q3) to the left and right of IP1 and IP5 [8].

Following the standard matching procedure [9] with MADX [10], using as variables the strengths of the quadrupoles in IR1 and IR5, achieves a minimum value of $\beta^{*}$ in IP1 and IP5 respectively of about $30 \mathrm{~cm}$. At this point the mechanical acceptance of most of the magnets in the matching section and the gradients of the matching quadrupoles reach their limits. Furthermore, the high $\beta$ functions in the IT generate huge chromatic aberrations that exceed the capabilities of the standard chromatic correction scheme performed by the sextupole families [9].

In order to further reduce the $\beta^{*}$ the achromatic telescopic squeezing (ATS) scheme [9] has been proposed to overcome the previous limitations as explained below.

\section{B. Basic principles of the ATS}

A standard matching procedure is done in the interaction regions to get a $\beta^{*}$ of $44 \mathrm{~cm}$ (named presqueezed $\beta^{*}$ ) in the case of round beams optics.

A further reduction of the presqueezed $\beta^{*}$ is followed by a telescopic squeeze, in which $\beta$-beating waves are generated in the arcs adjacent to the high luminosity insertions, namely the arc sectors $45,56,81$, and 12 , by adjusting the quadrupole strengths in the neighboring straight sections, i.e. IR4, IR6, IR8 and IR2.

A fundamental merit of this scheme is the locality of the chromaticity correction of the inner triplet by using one single arc of sextupoles on either side of IP1 and IP5. By adjusting the phase advance in the arc cell to $\pi / 2$, the $\beta$ waves generated in the arc reach their maximum at every other sextupole and the rate of increase of the $\beta$ function is proportional to the rate of decrease of the $\beta^{*}$ during the squeeze. This increase of the $\beta$ function in the location of these sextupoles enhances the efficiency of the chromaticity correction to compensate the chromaticity produced by the high $\beta$ functions in the IT.

This correction of the chromaticity also features the control of the chromatic Twiss parameters. This is studied via the Montague functions which are given by

$$
W=\frac{1}{2} \sqrt{A^{2}+B^{2}},
$$

where

$$
\begin{gathered}
A=\frac{1}{\beta} \frac{\partial \beta}{\partial \delta_{p}}, \\
B=\frac{\partial \alpha}{\partial \delta_{p}}-\alpha A
\end{gathered}
$$

and $\delta_{p}$ is the relative momentum deviation.

Minimizing the Montague functions in the insertions IR3 and IR7 is required to not deteriorate the collimation efficiencies.

The Montague functions have been studied previously for the LHC in [11] and the HL-LHC in [9], where further explanations about the overall ATS implementation can also be found.

The implementation of the ATS scheme provides a solid ground for the HL-LHC target of $\beta^{*}=15 \mathrm{~cm}$ in IP1 and IP5. Furthermore, the ATS scheme has also inspired a telescopic squeeze implemented in the Relativistic Heavy 
TABLE I. L-R LHeC baseline and possible upgrade parameters.

\begin{tabular}{|c|c|c|c|c|}
\hline & \multicolumn{2}{|c|}{ Baseline } & \multicolumn{2}{|c|}{ Upgrade } \\
\hline & Protons & Electrons & Protons & Electrons \\
\hline Luminosity $\left[10^{33} \mathrm{~cm}^{-2} \mathrm{~s}^{-1}\right]$ & 1 & 1 & 10 & 10 \\
\hline Beam energy $(\mathrm{GeV})$ & 7000 & 60 & 7000 & 60 \\
\hline Normalized emittance $\gamma \epsilon_{x, y}(\mu \mathrm{m})$ & 3.75 & 50 & 2.5 & 20 \\
\hline$\beta^{*}(\mathrm{~m})$ & 0.1 & 0.12 & 0.05 & 0.10 \\
\hline rms beam size $\sigma_{x, y}(\mu \mathrm{m})$ & 7 & 7 & 4 & 4 \\
\hline rms beam divergence $\sigma_{x, y}(\mu \mathrm{rad})$ & 70 & 58 & 80 & 40 \\
\hline Beam current $(\mathrm{mA})$ & $430(860)$ & 6.4 & 1112 & 25 \\
\hline Bunch spacing (ns) & $25(50)$ & $25(50)$ & 25 & 25 \\
\hline Bunch population $\left[10^{9}\right]$ & $1.7 \times 10^{2}$ & $1(2)$ & $2.2 \times 10^{2}$ & 4 \\
\hline Bunch charge (nC) & 27 & $0.16(0.32)$ & 35 & 0.64 \\
\hline
\end{tabular}

Ion Collider (RHIC) to further reduce the $\beta^{*}$ [12]. The following sections explain the $\mathrm{LHeC}$ design to later on extend the ATS scheme to include the $\mathrm{LHeC}$ insertion.

\section{LHeC}

The linac-ring option was presented in the LHeC CDR [13]. Construction of an energy recovery linac has been proposed to accelerate the electron beam up to $60 \mathrm{GeV}$ and collide it with the $7 \mathrm{TeV}$ LHC proton beam at IP2 to achieve collisions with a luminosity of $10^{33} \mathrm{~cm}^{-2} \mathrm{~s}^{-1}$. The baseline design parameters together with the possible upgrade towards a higher luminosity of $10^{34} \mathrm{~cm}^{-2} \mathrm{~s}^{-1}$ are shown in Table I.

\section{A. Layout}

The design of the LHeC IR aims to focus the counterclockwise proton beam 2 and collide it with the electron beam while the clockwise proton beam 1 bypasses the interaction.

A first conceptual design of the LHeC linac-ring IR was presented in [14]. The merits of the IR are a very low $\beta^{*}$ of $0.1 \mathrm{~m}$ with proton triplets as close as possible to the interaction point (IP) to minimize chromaticity. Head-on proton-electron collisions are achieved by means of dipoles around the IP.

A crossing angle of $6.8 \mathrm{mrad}$ between the noncolliding proton beams allows enough separation to place the proton IT. Only the proton beam colliding with the electrons is focused. A possible configuration in IR2 could be to inject the electrons parallel to the LHC beam 1 and collide them head-on with beam 2 as shown in Fig. 2.

The polarity and strengths of the separation and recombination dipoles (D1 and D2) have to be changed to allow for the large crossing angle at the IP. The new D1 has one aperture per beam and it is stronger than the nominal LHC by a factor of 3.43 . The new D2 is stronger than the nominal LHC by a factor of 1.21 . Both dipoles require a field of about $6 \mathrm{~T}$. The lengths of the D1 and D2 dipoles have been left unchanged and equal to $9 \mathrm{~m}$. However the final IR design will need to incorporate an escape line for the neutral particles coming from the IP, probably requiring to split D1 into two parts separated by tens of meters.

Bending dipoles around the IP are used to make the electrons collide head-on with beam 2 and to safely extract the disrupted electron beam. The required field of these dipoles is determined by the distance to the inner triplet $\left(L^{*}\right)$ and the minimum separation of the electron and the focused beam at the first quadrupole (Q1). A $0.3 \mathrm{~T}$ field extending over $9 \mathrm{~m}$ allows for a beam's separation of $0.075 \mathrm{~m}$ at the entry of Q1. This separation distance is compatible with mirror quadrupole design using $\mathrm{Nb}_{3} \mathrm{Sn}$ technology as illustrated in Fig. 3. The electron beam radiates $49 \mathrm{~kW}$ in the IR dipoles. A sketch of the beam trajectories, the synchrotron radiation fan and the proton triplets is shown in Fig. 4.

\section{B. Optics}

The $\mathrm{LHeC}$ interaction region is equipped with two special triplets at a distance $L^{*}$ of $10 \mathrm{~m}$ from the IP.

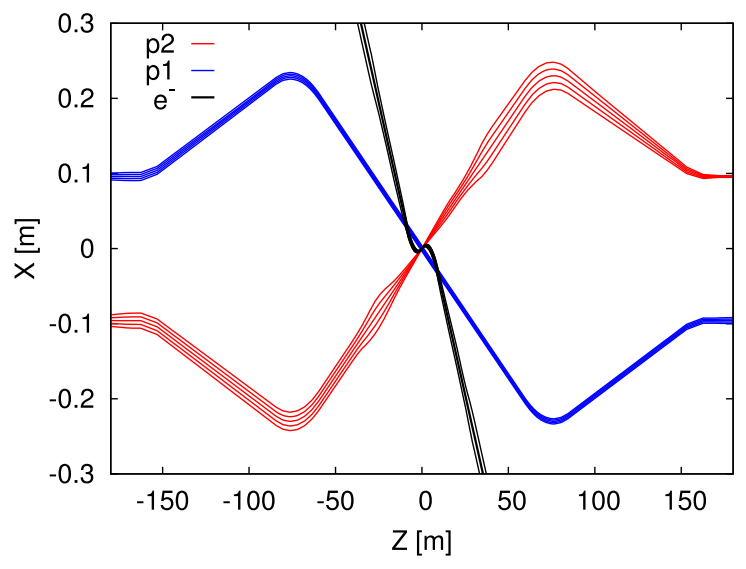

FIG. 2. Schematic view of the trajectories dictated by the corresponding recombination dipoles of proton beams 1 and 2 (blue and red respectively), and the electron beam (black) in IR2 with $5 \sigma$ and $10 \sigma$ envelopes [15]. 

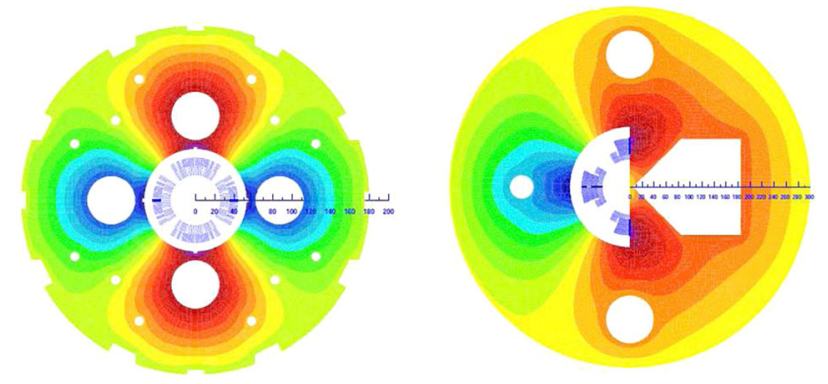

FIG. 3. Design of the half quadrupole for Q1 (right) and the normal quadrupole for Q2 (left). These magnets allowed a separation between the proton beams of $68 \mathrm{~mm}$ for the half quadrupole and of $87 \mathrm{~mm}$ for the normal quadrupole [13].

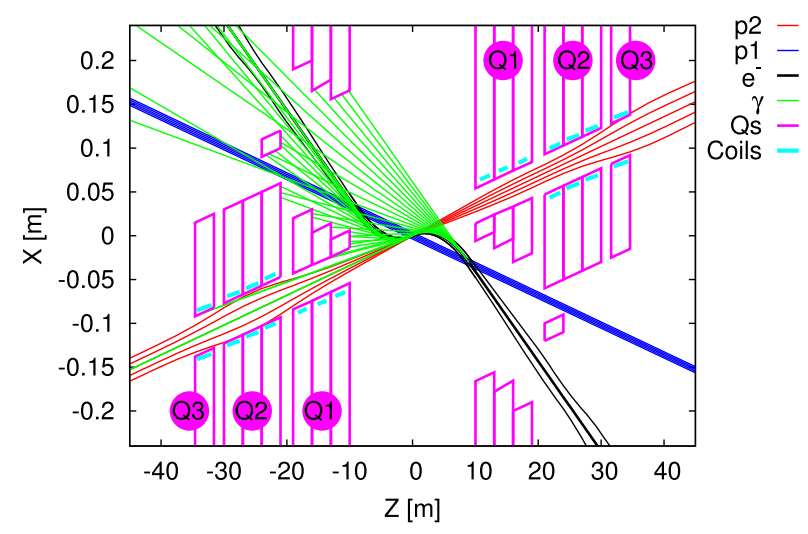

FIG. 4. Focused proton beam 2 (red) colliding with electron beam (black) while unfocused proton beam 1 bypasses the interaction. Each proton and electron beam passes through its corresponding aperture in the inner triplet [15].

Each triplet consists of three quadrupoles. The design of these quadrupoles includes a free field aperture for the proton beam 1 and the electron beam to pass through.

It was initially hoped that a compact $\mathrm{Nb}_{3} \mathrm{Sn}$ triplet with $L^{*}=10 \mathrm{~m}$ would allow for a normal chromaticity correction using the arc sextupoles. However after matching this triplet to the LHC and correcting linear chromaticity the chromatic $\beta$-beating at $d p / p=0.001$ is about $100 \%$. This is intolerable regarding collimation and machine protection issues [13].

The challenge at this point relied in achieving an optics that would not only achieve the $\beta^{*}=10 \mathrm{~cm}$ while maintaining the HL-LHC insertions undisturbed, but that would also provide a dedicated chromaticity correction scheme.

\section{ATS EXTENSION TO THE LHeC}

The implementation of the ATS scheme into the LHeC IR presents an extra challenge as the LHeC IR is adjacent to the HL-LHC insertion IR1 and the ATS, as explained previously, makes use of the adjacent regions for the telescopic squeeze. This further difficulty adds a constraint

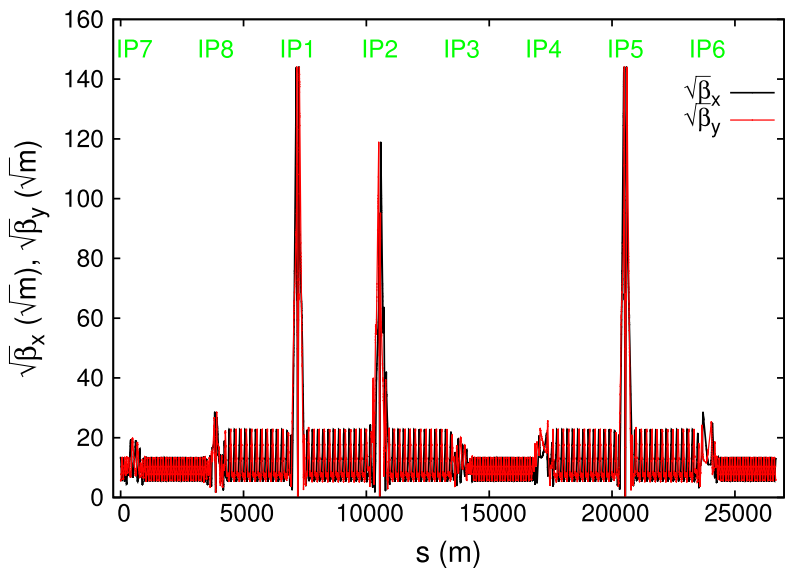

FIG. 5. LHeC ATS collision optics for beam 2 with $\beta^{*}=10 \mathrm{~cm}$ at IP2 and $\beta^{*}=15 \mathrm{~cm}$ at IP1 and IP5.

to be taken into account to achieve the reduction of the $\beta^{*}$ in the three interaction points.

Following a proposal to integrate the $\mathrm{LHeC}$ IR into the HL-LHC lattice using the ATS scheme [16], a first study of the required proton optics for the nominal case with $\beta^{*}=10 \mathrm{~cm}$ and $L^{*}=10 \mathrm{~m}$ was presented [17] and it is further described in this section.

The proton optics in the LHeC IR with the new triplets was designed to extend the ATS scheme without modification of the HL-LHC optics [18] (version HLLHCv1.0 for round proton beams). In this procedure the arc cells in sector 23 are exactly adjusted to the phase advance of $\pi / 2$. Then the ATS matching conditions for proton beam 2 are imposed for the left and right phase advance of IR2 (with respect to IP2). The LHeC IR provides a $\beta^{*}$ of about $30 \mathrm{~cm}$ at IP2 for beam 2 with no beta beating in the arcs (presqueezed optics). The transition from the presqueezed to the collision optics is performed by a telescopic squeeze using the quadrupoles in IR8, IR3, IR4 and IR6 with no variation of the quadrupole strengths in IR1, IR2 and IR5 at this stage. During the transition $\beta$ waves in sectors 45,56 , 81,12 , and 23 start to build up producing further reductions in $\beta^{*}$ from about 30 to $10 \mathrm{~cm}$ at IP2 and from 44 to $15 \mathrm{~cm}$ at IP1 and IP5 as shown in Fig. 5. The peaks of the $\beta$ functions occur in the inner triplets.

\section{FLEXIBILITY OF THE IR DESIGN}

The nominal design provides a solution to obtain a $\beta^{*}$ of $10 \mathrm{~cm}$ at IP2 for proton beam 2 with the aid of a new IT at $L^{*}=10 \mathrm{~m}$. The flexibility of this design is of great interest because of the benefits that could be obtained in terms of synchrotron radiation power and luminosity.

This flexibility is studied via two methods. The first one is exploring the minimization of $\beta^{*}$, to obtain an upper limit of the luminosity. The second one is by increasing $L^{*}$, which would result in a minimization of synchrotron radiation power, since it requires less bending to steer 
the $e^{-}$beam into the field free aperture of the proton inner triplet.

Keeping the optics parameters at the ends of the IR2 fixed, the strengths of the quadrupoles in the IR2 can be used as variables to find solutions for different values of $L^{*}$ and $\beta^{*}$.

The matched solutions for the optics with $L^{*}=$ $\{10,11,12,13,14,15,16,17,18,19,20\} \mathrm{m}$ and $\beta^{*}$ fixed at $10 \mathrm{~cm}$ have been found, as well as for the optics with $\beta^{*}=\{5,6,7,8,9,10,20\} \mathrm{cm}$ with a fixed $L^{*}$ at $10 \mathrm{~m}$.

The cases with $L^{*}>14 \mathrm{~m}$ are in particular interesting because, first, they generate less synchrotron radiation power and second because at this distance the separation between the beams is enough ( $\geq 87 \mathrm{~mm}$ ) to be able to use a normal quadrupole (left side of Fig. 3) as opposed to the half quadrupole design (right side of Fig. 3). Stray fields are found in the field free regions [19] of this half quadrupole, increasing the difficulty to match the electron beam lattice [20].

\section{CHROMATICITY CORRECTION}

In the previous section, different lattice configurations were found in order to provide solutions for different $L^{*}$ and $\beta^{*}$. However, careful studies for chromaticity correction is still needed to be done to validate the extended ATS scheme for the $\mathrm{LHeC}$ lattice.

The design of the IRs should meet a lot of different criteria and some disadvantages arise if the $\beta^{*}$ and $L^{*}$ parameters are changed.

Minimizing $\beta^{*}$ and increasing $L^{*}$ both result in an increase of the peak $\beta$ function in the location of the inner triplet, and hence an increase in the chromatic aberrations.

The natural chromaticity is shown as a function of $\beta^{*}$ in Fig. 6 and as a function of $L^{*}$ in Fig. 7. As it can be seen in the figures, the absolute magnitude of the natural chromaticity increases linearly as $L^{*}$ grows, whereas minimizing $\beta^{*}$ causes the absolute magnitude of the natural chromaticity to increase more rapidly.

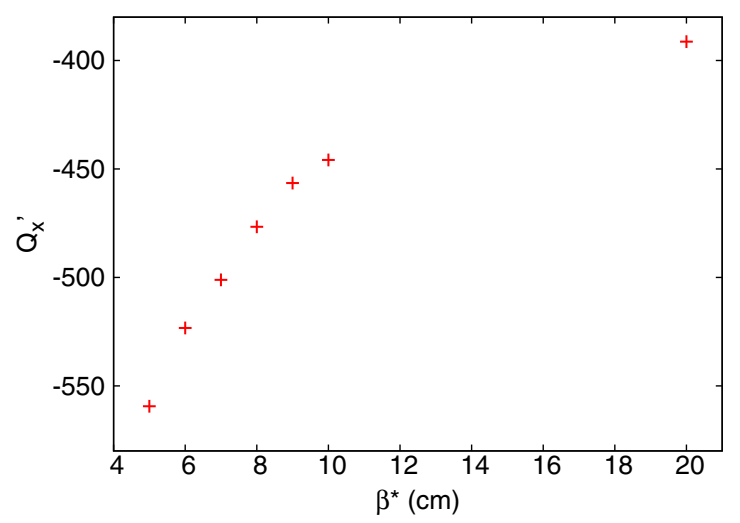

FIG. 6. Natural chromaticity plotted as a function of $\beta^{*}$ with $L^{*}$ fixed at $10 \mathrm{~m}$.

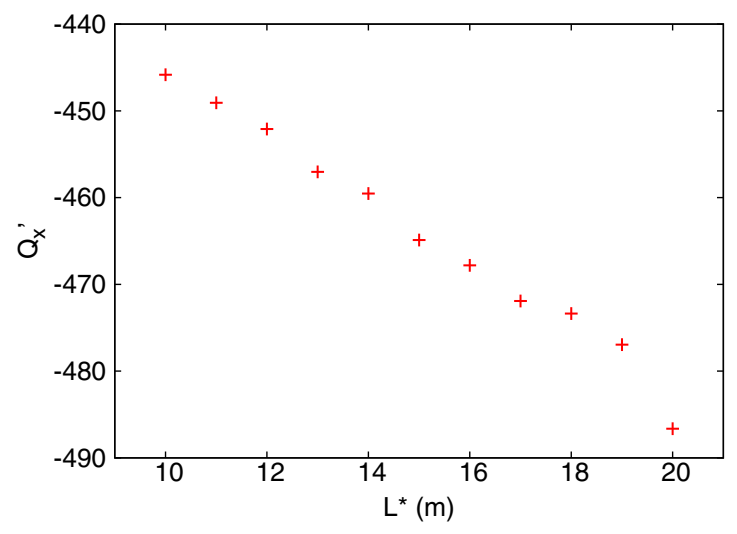

FIG. 7. Natural chromaticity plotted as a function of $L^{*}$ with $\beta^{*}$ fixed at $10 \mathrm{~cm}$.

In the following section, the chromaticity correction is presented for the nominal case $\left(L^{*}=10 \mathrm{~m}, \beta^{*}=10 \mathrm{~cm}\right)$, and the limits of the correction for cases with higher natural chromaticity are explored.

\section{A. Nominal case correction}

As it was described in Sec. II B, the chromaticity correction for the HL-LHC was achieved using only one family of sextupoles (in each horizontal and vertical plane) on both sides from the IPs. However, in the LHeC, an imbalance exists due to the $\beta$ wave produced to perform the telescopic squeeze in both IP1 and IP2. The path to follow is then trying to achieve a global correction that might break the locality of the chromatic correction but will certainly bring benefits in terms of controlling the chromatic aberrations.

The optimization of the strength of the sextupole families to achieve the control of the chromaticity is performed by a new developed matching procedure in MADX which takes as variables the strength of all the families of sextupoles acting independently, to fix as constraints the reduction of the horizontal $\left(Q_{x}^{\prime}\right)$ and vertical $\left(Q_{y}^{\prime}\right)$ chromaticities to a value of 2 and the reduction of the chromatic betatron amplitude function, in both transverse planes $\left(W_{x}\right.$ and $\left.W_{y}\right)$, below 200 in the collimation insertions IR3 and IR7.

This new procedure, named $\mathrm{LHeC}$-like, was performed successfully for the case $L^{*}=10 \mathrm{~m}$ and $\beta^{*}=10 \mathrm{~cm}$, where the natural chromaticities are $Q_{x}^{\prime}=-445.83$ and $Q_{y}^{\prime}=-446.77$. The Montague functions along the ring are shown in Fig. 8 where the adjustments to a value of 200 are observed in IR3 and IR7.

In order to provide a comparison, Fig. 9 shows the Montague functions $W_{x}$ and $W_{y}$ having done a LHC-like correction, which takes two variables, the focusing and defocusing sextupole families, to adjust as constraints $Q_{x}^{\prime}=Q_{y}^{\prime}=2$, leaving the Montague functions $W_{x, y}$ free to vary.

The merits of the $\mathrm{LHeC}$-like correction are clear in comparison with the LHC-like correction (Figs. 8 and 9), 


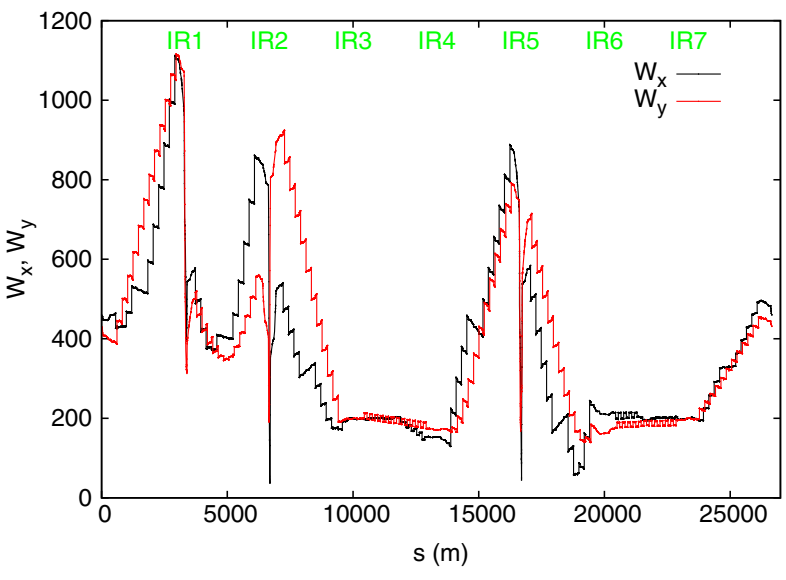

FIG. 8. Montague $W_{x}$ and $W_{y}$ functions along beam 2 after the LHeC-like chromatic correction (the machine starts in IP8).

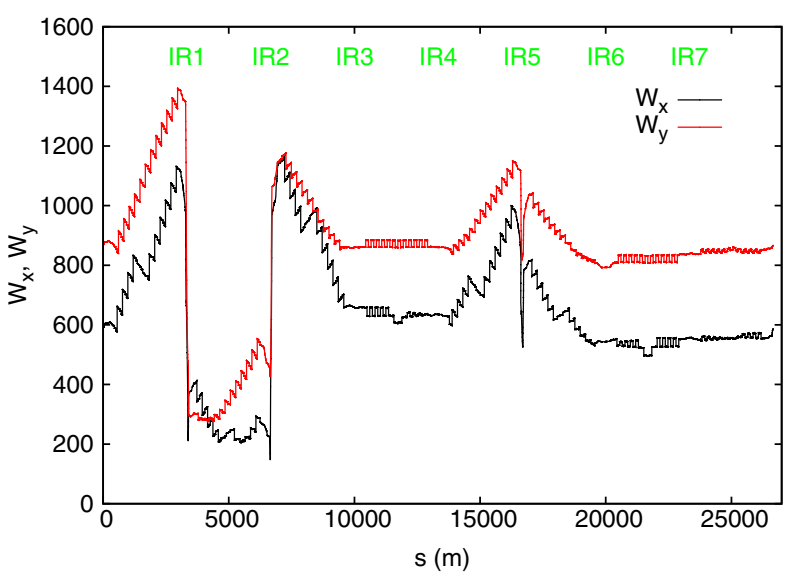

FIG. 9. Montague $W_{x}$ and $W_{y}$ functions after the LHC-like chromatic correction along beam 2 (the machine starts in IP8).

not only because the Montague functions are controlled in the collimation insertions IR3 and IR7, but also the overall Montague functions are smaller along the ring, including a $20 \%$ reduction of the Montague peak functions.

The chromatic variation of the betatron tunes over a momentum range of $\delta p= \pm 0.001$ has also been studied. Figure 10 shows the horizontal betatron tune $Q_{x}$ as a function of $\delta_{p}$ for the two corrections LHC-like and LHeClike. Figure 11 shows the deviation of the betatron tunes $Q_{x}$ and $Q_{y}$ from the working point for both corrections plotted on the resonance map up to the 10th order within the range of $\delta_{p}= \pm 0.001$.

The presence of nonlinearities are clearly observable in both cases, but the $\mathrm{LHeC}$-like correction is able to avoid resonances up to the 10th order, while LHC-like fails to do so. Frequency map analysis and tracking studies for the dynamic aperture are expected to provide more information about the influence of the nonlinearities and different resonances on the beam dynamics (see Sec. VII).

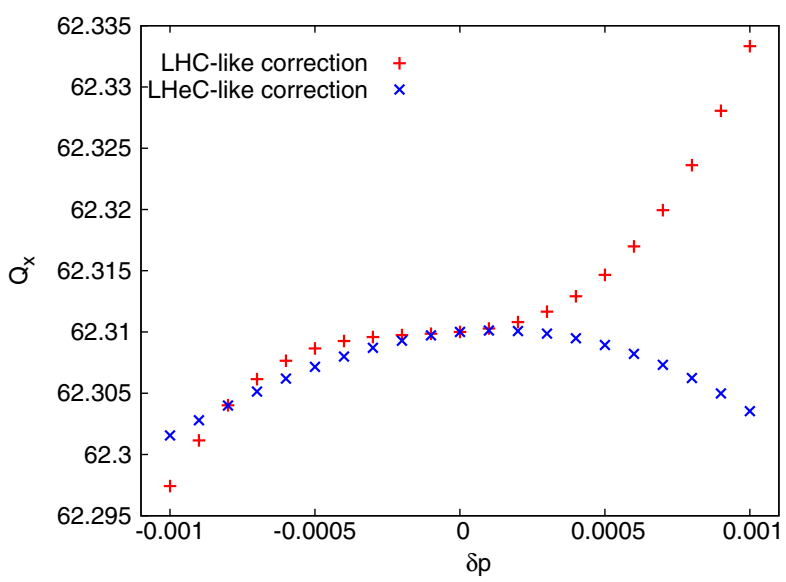

FIG. 10. Horizontal betatron tune $Q_{x}$ vs $\delta_{p}$ after the LHC-like and LHeC-like chromaticity corrections.

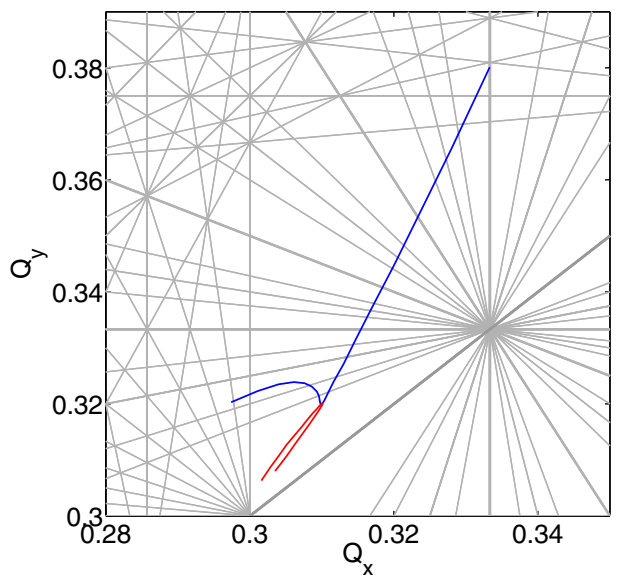

FIG. 11. Horizontal $\left(Q_{x}\right)$ and vertical $\left(Q_{y}\right)$ betatron tunes within the momentum range $\delta p= \pm 0.001$ for beam 2 plotted on the resonance map up to the 10th order for the LHC-like (blue) and LHeC-like (red) chromaticity corrections.

\section{B. Chromaticity correction limits}

The LHeC-like chromaticity correction procedure in MADX was performed to check the chromaticity correction in the cases for different $L^{*}$ and $\beta^{*}$, found in Sec. IV, to identify the maximum chromaticity that can be corrected with this method.

Successful LHeC-like chromaticity correction $\left(W_{x, y}<200\right.$ in IR3 and IR7, $\left.Q_{x}^{\prime}=Q_{y}^{\prime}=2\right)$ in which the betatron tune spread avoids any resonances until 9th order is achieved for the optics with $8 \mathrm{~cm} \leq \beta^{*} \leq 10 \mathrm{~cm}$ at fixed $L^{*}=10 \mathrm{~m}$, and $10 \mathrm{~m} \leq L^{*} \leq 18 \mathrm{~m}$ with constant $\beta^{*}$ of $10 \mathrm{~cm}$. The $\mathrm{LHeC}$-like correction procedure fails to converge outside the obtained limits of the $\beta^{*}$ and $L^{*}$. Considering the natural chromaticity shown in Figs. 6 and 7 we can draw the limit of the chromaticity correction by this method for cases with natural chromaticity below $\approx-480$ as illustrated in Fig. 12. 


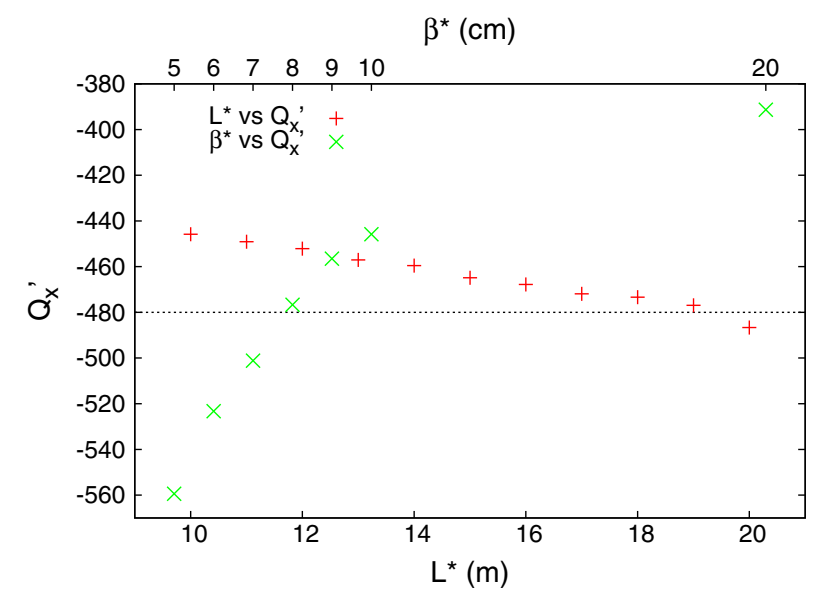

FIG. 12. Limit of the chromatic correction (black dashed line) overlaid in the plot $Q_{x}^{\prime}$ vs $L^{*}$ (red) and $Q_{x}^{\prime}$ vs $\beta^{*}$ (green). This limit was found around $Q_{x}^{\prime} \approx-480$ for a maximum of $L^{*}=18 \mathrm{~m}$ with $\beta^{*}=10 \mathrm{~cm}$ and a minimum $\beta^{*}$ of $8 \mathrm{~cm}$ with $L^{*}=10 \mathrm{~m}$.

For the following sections we will consider the cases with $\beta^{*}=10 \mathrm{~cm}$ and $L^{*}>10 \mathrm{~m}$ since this value is enough to achieve the luminosity of $L=10^{33} \mathrm{~cm}^{-2} \mathrm{~s}^{-1}$ and we also need to explore ways for minimizing the synchrotron radiation, as explain in Sec. IV. However the case with $\beta^{*}=5 \mathrm{~cm}$ requires further study in order to explore the upgrade luminosity of $L=10^{34} \mathrm{~cm}^{-2} \mathrm{~s}^{-1}$ (Table I).

\section{SYNCHROTRON RADIATION}

The synchrotron radiation power produced by the electron beam while transporting it to and from the IP is a problem that has to be treated with great care in order to minimize the damage in the quadrupoles and the detector.

The synchrotron radiation power as a function of the circulating particle current (I), the energy of the beam (E) and the bending radius $\left(\rho_{e}\right)$ is given by [21]

$$
P[\mathrm{MW}]=8.85 \times 10^{-5}\left[\mathrm{~m} / \mathrm{GeV}^{3}\right] \frac{E^{4}[\mathrm{GeV}]}{\rho_{e}[\mathrm{~m}]} I[\mathrm{~A}] .
$$

Each dipole covers an arc of the ring subtended by an angle $\theta$, taking this into account for two dipoles Eq. (4) becomes:

$$
P[\mathrm{MW}]=8.85 \times 10^{-5}\left[\mathrm{~m} / \mathrm{GeV}^{3}\right] \frac{E^{4}[\mathrm{GeV}] \theta_{e}}{2 \pi \rho_{e}[\mathrm{~m}]} I[\mathrm{~A}] \times 2 .
$$

The radius and angle are given in Eqs. (6) and (7) respectively, as a function of the distance from the IP to the inner triplet $L^{*}$ and the distance from the normal aperture to the field free aperture $\Delta$ :

$$
\rho_{e}=\frac{L^{* 2}}{2 \Delta}
$$

$$
\theta_{e}=\arcsin \left(\frac{2 \Delta}{L^{*}}\right)
$$

From these equations is clear that increasing $L^{*}$ will result in an increase of the radius $\rho_{e}$ and a decrease of the angle $\theta_{e}$, both producing a reduction of the total synchrotron radiation power as previously reported [17].

This paper explores a possible way to reduce this power further via the reduction of the parameter $\Delta$ since this will result in a reduction of the synchrotron radiation power in the same way that increasing the $L^{*}$ did.

In order to find the minimum value for the separation $\Delta$ the following constraints are considered: (i) The distance between the beams has to be greater than $75 \mathrm{~mm}$ for $L^{*}<14 \mathrm{~m}$ (separation in half quadrupole design) and greater than $87 \mathrm{~mm}$ for $L^{*}>14 \mathrm{~m}$ (separation in normal quadrupole design). (ii) To avoid parasitic beam-beam interactions (long range encounters) the separation at the first long-range encounter has to be of at least $12 \sigma$ (with $\sigma$ being the rms beam size). This long range encounter corresponds to a distance from the IP2 of $s=3.75 \mathrm{~m}$ for a $25 \mathrm{~ns}$ bunch spacing and a distance $s=7.5 \mathrm{~m}$ for a $50 \mathrm{~ns}$ bunch spacing. (iii) The size of the proton and electron beam must physically fit inside the normal and field free apertures respectively.

Results show that given the minimum separation distance from the first constraint, the second one is automatically fulfilled, except for the case with $L^{*}=20 \mathrm{~m}$ and 25 ns bunch spacing in which case the second constraint gives the minimum separation. Table II shows the minimum distance for each $L^{*}$, along with the aperture in $\sigma$ of the electron and the proton beam 2 that could be fitted in the field free and normal aperture respectively, to make sure the third constraint is also fulfilled, and finally, the power of the synchrotron radiation it produces.

TABLE II. Minimum separation between beams $(\Delta)$ corresponding to each $L^{*}$ and bunch spacing. The number of sigmas

\begin{tabular}{|c|c|c|c|c|c|}
\hline$L^{*}$ & $\begin{array}{c}\text { Minimum } \\
\Delta_{25} \\
\end{array}$ & $\begin{array}{c}\text { Minimum } \\
\Delta_{50} \\
\end{array}$ & $\begin{array}{c}e \text {-beam } \\
(\sigma)\end{array}$ & $\begin{array}{c}p \text {-beam } \\
2(\sigma)\end{array}$ & $\begin{array}{c}P \\
(\mathrm{~kW})\end{array}$ \\
\hline 10 & 0.075 & 0.075 & 41 & 32 & 49.29 \\
\hline 11 & 0.075 & 0.075 & 38 & 29 & 37.03 \\
\hline 12 & 0.075 & 0.075 & 34 & 27 & 28.52 \\
\hline 13 & 0.075 & 0.075 & 32 & 24 & 22.43 \\
\hline 14 & 0.087 & 0.087 & 27 & 23 & 24.17 \\
\hline 15 & 0.087 & 0.087 & 25 & 21 & 19.65 \\
\hline 16 & 0.087 & 0.087 & 24 & 20 & 16.19 \\
\hline 17 & 0.087 & 0.087 & 22 & 19 & 13.50 \\
\hline 18 & 0.087 & 0.087 & 21 & 18 & 11.37 \\
\hline 19 & 0.087 & 0.087 & 20 & 17 & 9.67 \\
\hline 20 & 0.091 & 0.087 & 19 & 16 & 8.29 \\
\hline
\end{tabular}
that can be fitted inside the corresponding aperture for the electron and proton beam is also shown, along with the synchrotron radiation power it produces. 


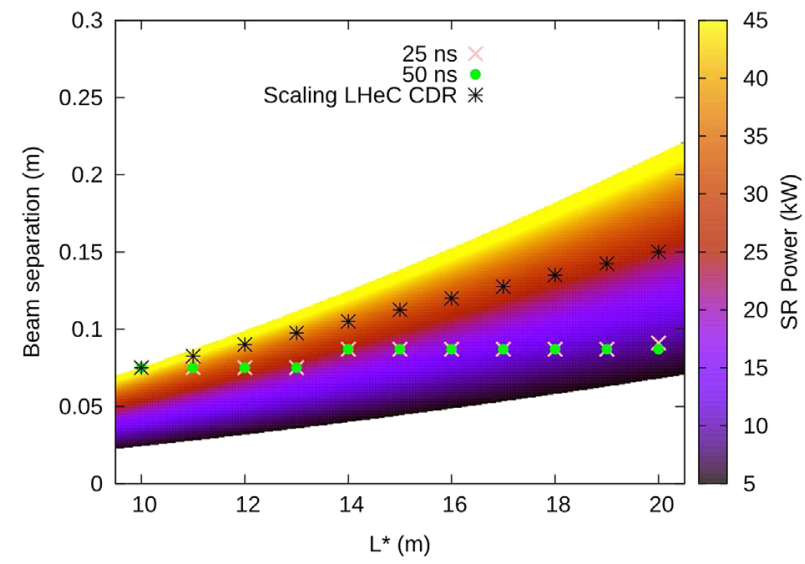

FIG. 13. Synchrotron radiation power given as a function of $L^{*}$ and the beam separation in Q1. The black symbols show the cases for scaling the LHeC CDR, the pink and green symbols (almost overlaid in the image) show the minimum beam separation for bunch spacings 25 and 50 ns respectively.

Figure 13 shows the radiation power as a function of $L^{*}$ and the beam separation for three cases: the first case illustrates the results of scaling the $\mathrm{LHeC}$ CDR reported in [17], the second and third cases illustrate the minimum distance for the 25 and $50 \mathrm{~ns}$ bunch spacings. The reduction of the synchrotron radiation power for the cases $L^{*}>10 \mathrm{~m}$ is clearly observed.

In order to reduce the amount of synchrotron radiation hitting the inner triplet a new interesting option with "sweet spot" magnets [22] is being considered. The possible integration of this new magnet design may give additional flexibility to the final lattice design.

\section{DYNAMIC APERTURE STUDIES}

The dynamic aperture (DA), i.e., the largest amplitude of the domain in phase space where the particle motion is stable, has been used as a key parameter to specify the machine performance since the design phase of the LHC [23].

In this section, DA studies are performed in order to quantify the impact of nonlinearities (mainly produced by the strong sextupoles) and validate the $\mathrm{LHeC}$ lattice.

The DA was computed by the particle tracking code SIXTRACK [24] over $10^{5}$ turns, using a polar grid of initial conditions distributed in order to have 30 particles for each $2 \sigma$ interval. Five different values of phase angle have been used. The momentum offset is set to $2.7 \times 10^{-4}$ and the value of the normalized emittance is $\epsilon=3.75 \mu \mathrm{m}$.

To account for the magnetic imperfections, 60 different realizations (seeds) of the errors for the LHC magnets have been assigned. These 60 combinations were produced using the program WISE [25], which incorporates all known uncertainties in the magnet error measurement. At the moment, the errors of the new IT and recombination dipoles D1 and D2 for IR1, IR2 and IR5 have not been considered,

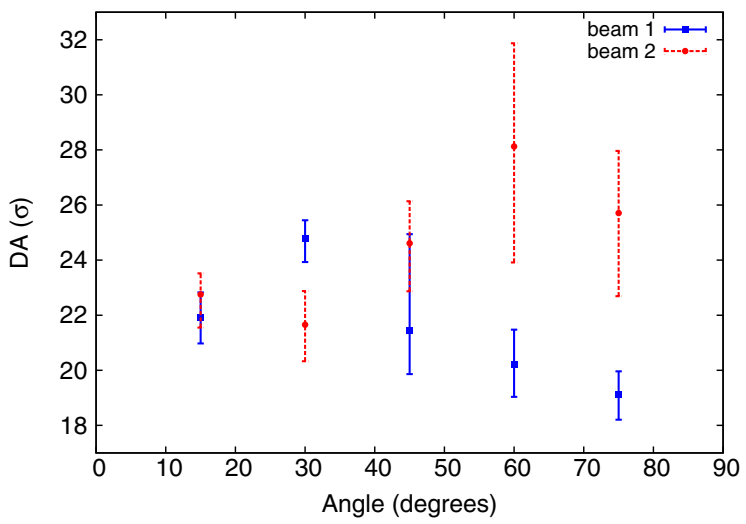

FIG. 14. Comparison between DA of beam 1 and beam 2 for the round optics of the HLLHCV1.0 version of the HL-LHC experiment $\left(\beta_{\mathrm{IP} 1,5}=15 \mathrm{~cm}\right.$ and $\left.\beta_{\mathrm{IP} 2}=10 \mathrm{~m}\right)$ without errors for IT, Q4, Q5, D1 and D2 of IR1 and IR5.

as well as the errors for the additional quadrupoles Q4, Q5 for the HL insertions IR1 and IR5, since at the time of this paper the values of these errors remained under study. The correction techniques for the IR nonlinearities are given in [26,27].

Studies have already been carried out for the previous lattice version of the HL-LHC (SLHCV3.1b) for beam 1 [28]. The DA is expected to be different for beam 1 and beam 2 , so a comparison was made between the two beams for the round optics $\left(\beta_{\mathrm{IP} 1,5}=15 \mathrm{~cm}\right.$ and $\left.\beta_{I P 2}=10 \mathrm{~m}\right)$ of the HLLHCV1.0 lattice as shown in Fig. 14.

Figure 15 represents the DA of the HL-LHC with round optics for beam 2 in comparison to the HL-LHC optics with the integrated $\mathrm{LHeC}$ IR $\left(\beta_{I P 1,5}=15 \mathrm{~cm}\right.$ and $\beta_{I P 2}=10 \mathrm{~cm}$ ) for the nominal case with $L^{*}=10 \mathrm{~m}$. The sextupole families for the HL-LHC + LHeC optics are stronger than for the HL-LHC optics to compensate a big amount of chromaticity contributed by the LHeC IR that causes a clear reduction of the DA.

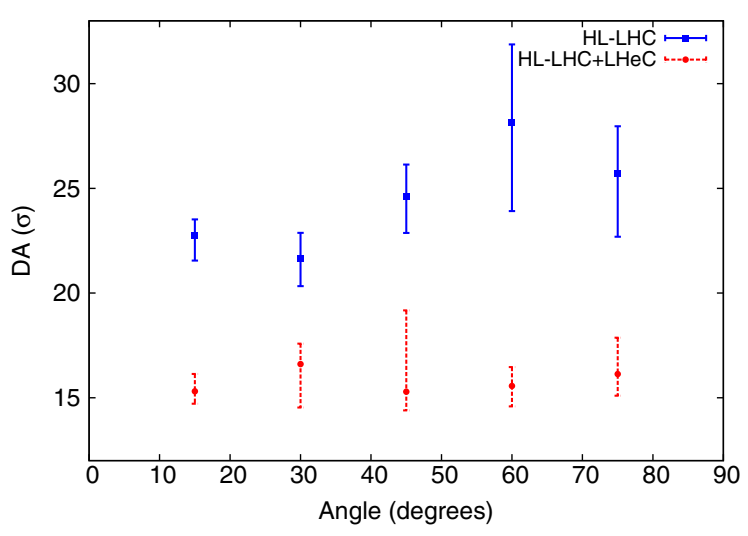

FIG. 15. Comparison of beam 2 DA between the round optics of the HLLHCV1.0 version of the HL-LHC $\left(\beta_{\mathrm{IP} 1,5}=15 \mathrm{~cm}\right)$ and with the LHeC insertion with $\beta^{*}=10 \mathrm{~cm}$ and $L^{*}=10 \mathrm{~m}$ in IP2. 


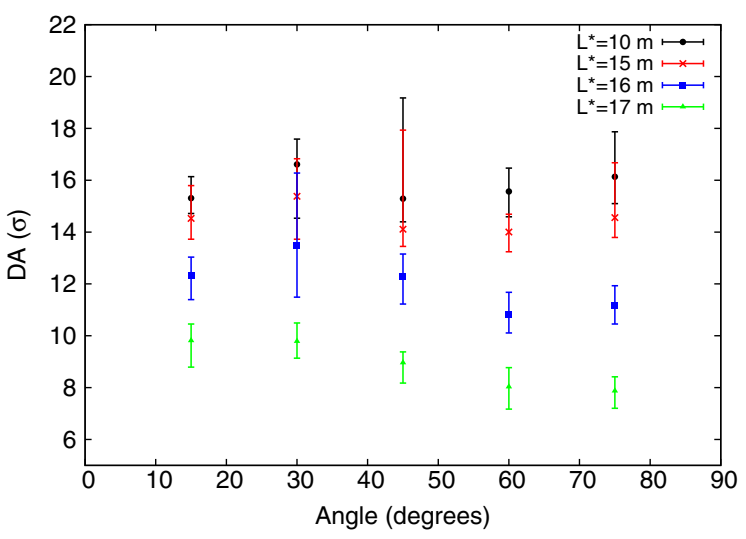

FIG. 16. Comparison of DA studies for different values of $L^{*}$. Cases with $L^{*}=10,15,16,17 \mathrm{~m}$ with a fixed $\beta^{*}=10 \mathrm{~cm}$ are shown.

Figure 16 represents the comparison between the cases $L^{*}=10,15,16$ and $17 \mathrm{~m}$. A small reduction of DA is observed for the case $L^{*}=15 \mathrm{~m}$ but it is still very close to the DA found for $L^{*}=10 \mathrm{~m}$. However, from this value on, the DA reduction decreases more rapidly as we can observe for the cases $L^{*}=16 \mathrm{~m}$ and $L^{*}=17 \mathrm{~m}$.

This paper suggests that the case with $L^{*}=15 \mathrm{~m}$ and $\beta^{*}=10 \mathrm{~cm}$ features the largest possible $L^{*}$ regarding long term stability. However, further studies are to be continued with the inclusion of the errors of the inner triplets in the three interaction points in order to maintain the minimum DA required to ensure the stability of the beam.

\section{FREQUENCY MAP ANALYSIS}

The frequency map analysis (FMA) has been applied in the LHC in order to study its nonlinear dynamics [29]. This method relies in the high precision calculation of the frequencies of motion. By studying the behavior of the tunes associated with a sample of initial conditions, it is possible to obtain some indication of the stability of a system [30].

The FMA is performed by the program SUSSIX [31] and applied to the short term tracking in SIXTRACK $\left(10^{3}\right.$ turns) for a sample of initial amplitudes $\left.I=\sqrt{(} I_{x}^{2}+I_{y}^{2}\right)$ (from $0-22 \sigma)$ and angles $\arctan \left(I_{y 0} / I_{x 0}\right)$ (from 0-90 degrees).

The variation of these tunes is studied by calculating the diffusion factor, difference between the calculated tune at 5,000 and 10,000 turns:

$$
D=\log _{10} \sqrt{\left(\Delta Q_{x}\right)^{2}+\left(\Delta Q_{y}\right)^{2}} .
$$

The diffusion factor in a frequency map of order 12 is represented in Fig. 17 for the lattice with $L^{*}=10 \mathrm{~m}$ and $\beta^{*}=10 \mathrm{~cm}$ and in Fig. 18 for the lattice with $L^{*}=17 \mathrm{~m}$ and $\beta^{*}=10 \mathrm{~cm}$. These frequency maps also show the resonance lines $m_{x} Q_{x}+m_{y} Q_{y}=l$ where $m_{x}, m_{y}$ and $l$ are

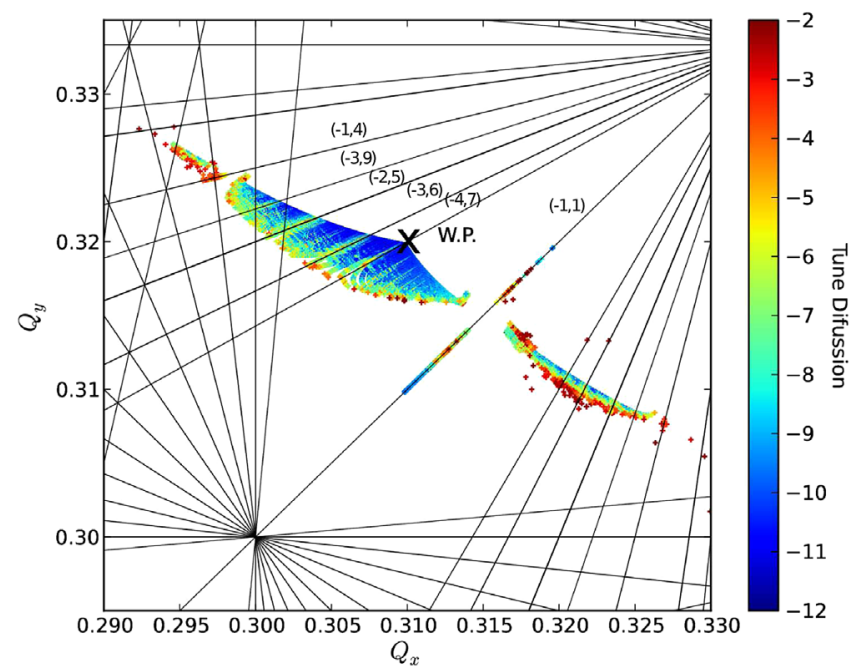

FIG. 17. Diffusion factor on a tune map for the initial angles varying from 0-90 degrees and initial amplitudes $I=0-22 \sigma$ over a resonance diagram of order 12 for the lattice with $L^{*}=10 \mathrm{~m}$ and $\beta^{*}=10 \mathrm{~cm}$.

integers. The resonance lines causing a disruption in the diffusion factor are labeled in the figure.

This diffusion factor is plotted also for the different initial amplitudes and angles for the lattice with $L^{*}=10 \mathrm{~m}$ and $\beta^{*}=10 \mathrm{~cm}$ in Fig. 19 , for the lattice with $L^{*}=15 \mathrm{~m}$ and $\beta^{*}=10 \mathrm{~cm}$ in Fig. 20 and for the lattice $L^{*}=17 \mathrm{~m}$ and $\beta^{*}=10 \mathrm{~cm}$ in Fig. 21. By analyzing these plots with the corresponding tune maps (Figs. 17 and 18) it is also possible to identify the resonance lines causing the disruption in the diffusion factor, now more explicit in terms of amplitude and angle, and are also shown in the figures.

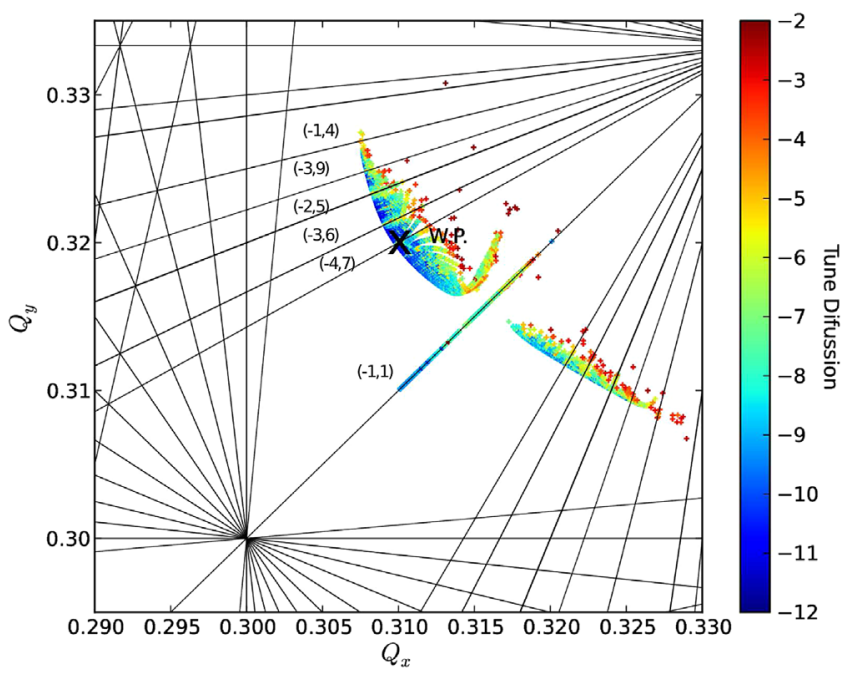

FIG. 18. Diffusion factor on a tune map for the initial angles varying from 0-90 degrees and initial amplitudes $I=0-22 \sigma$ over a resonance diagram of order 12 for the lattice with $L^{*}=17 \mathrm{~m}$ and $\beta^{*}=10 \mathrm{~cm}$. 


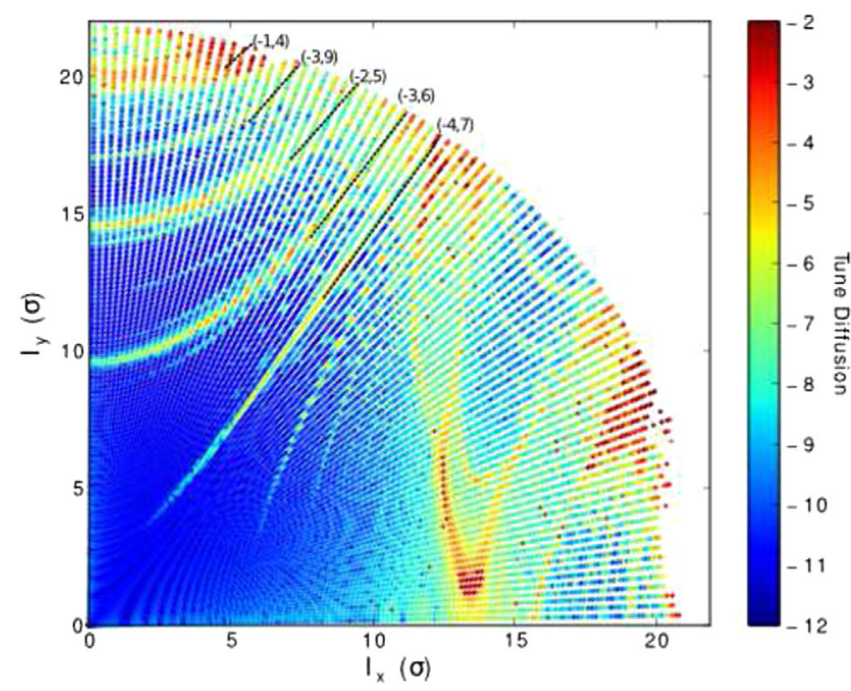

FIG. 19. Diffusion factor $D$ over the initial amplitudes $I=0-22 \sigma$ and 90 initial angles for the lattice $L^{*}=10 \mathrm{~m}$ and $\beta^{*}=10 \mathrm{~cm}$.

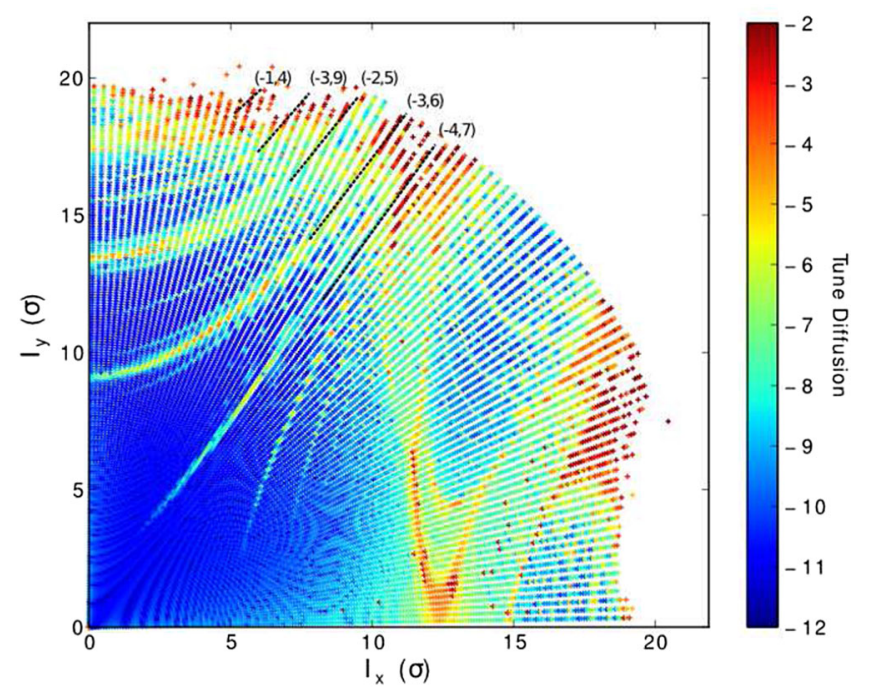

FIG. 20. Diffusion factor $D$ over the initial amplitudes $I=0-22 \sigma$ and 90 initial angles for the lattice $L^{*}=15 \mathrm{~m}$ and $\beta^{*}=10 \mathrm{~cm}$.

It is observed that the lattices with $L^{*}=10 \mathrm{~m}$ and $L^{*}=$ $15 \mathrm{~m}$ both with $\beta^{*}=10 \mathrm{~cm}$, present a similar behavior, except for the outer zones where the initial amplitudes $I$ are closer to $20 \sigma$, however these amplitudes are already larger than the dynamic aperture calculated over $10^{5}$ turns. Both lattices present areas with a larger diffusion factor at lower angles and initial amplitudes close to $12 \sigma$. On the other side, the diffusion factor is larger for angles 2246 degrees, the same region where larger dynamic aperture was observed in Fig. 16.

A clear reduction of the stable area of motion is observed for the lattice with $L^{*}=17 \mathrm{~m}$ and $\beta^{*}=10 \mathrm{~cm}$ as seen in Fig. 21. This is consistent with the DA results shown in Fig. 16. This configuration is at the limit where the

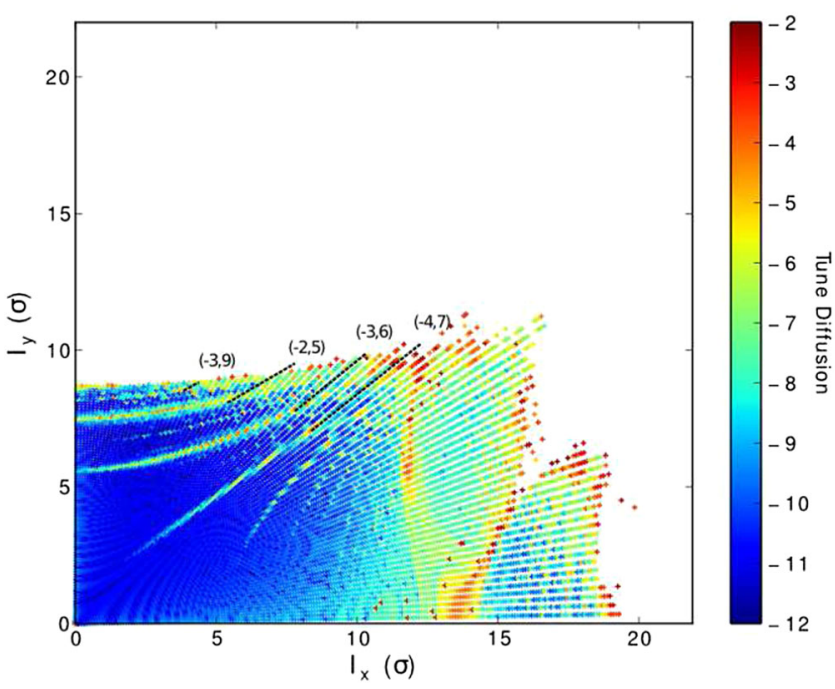

FIG. 21. Diffusion factor $D$ over the initial amplitudes $I=0-22 \sigma$ and 90 initial angles for the lattice $L^{*}=17 \mathrm{~m}$ and $\beta^{*}=10 \mathrm{~cm}$.

chromaticity can be successfully controlled with the sextupole correction scheme (see Fig. 12).

\section{CONCLUSIONS}

The proposed design for the $\mathrm{LHeC}$ IR to achieve e-p collisions with the required luminosity of $L=10^{33} \mathrm{~cm}^{-2} \mathrm{~s}^{-1}$ has been developed and integrated within the HL-LHC optics by extending the ATS scheme.

Different lattice configurations open up different possibilities for increasing $L^{*}$ up to $20 \mathrm{~m}$, and minimizing $\beta^{*}$ to $5 \mathrm{~cm}$. Chromaticity correction with the specified constraints on $Q_{x, y}$ and the Montague functions $W_{x, y}$ is only achieved up to $L^{*}=18 \mathrm{~m}$ with $\beta^{*}=10 \mathrm{~cm}$ and $\beta^{*}=8 \mathrm{~cm}$ with $L^{*}=10 \mathrm{~m}$. Tracking studies for the DA in the presence of different field and alignment errors show that the reasonable dynamic aperture is achieved for up to $L^{*}=15 \mathrm{~m}$ but significant reduction of DA is observed for $L^{*}>15 \mathrm{~m}$. Furthermore, frequency map analysis shows that the two different lattices $L^{*}=10 \mathrm{~m}$ with $\beta^{*}=10 \mathrm{~cm}$ and $L^{*}=15 \mathrm{~m}$ with $\beta^{*}=10 \mathrm{~cm}$ present similar behavior of the tune diffusion, but again several differences arise for the case with $L^{*}=17 \mathrm{~m}$ with $\beta^{*}=10 \mathrm{~cm}$. A maximum of $L^{*}=15 \mathrm{~m}$ is therefore recommended for the LHeC.

The complete study shows that the solution with $\beta^{*}=10 \mathrm{~cm}$ is enough to achieve the desired luminosity of the baseline version, but considerable benefits arise for the cases $L^{*}>10 \mathrm{~m}$, in particular for $L^{*}=15 \mathrm{~m}$. For this value of $L^{*}$, normal quadrupoles can be used, the chromaticity is controlled, there is a minimization of synchrotron radiation power, and the DA reduction is minimal with respect to the nominal case.

The more challenging parameters of the upgrade version to achieve a luminosity of $L=10^{34} \mathrm{~cm}^{-2} \mathrm{~s}^{-1}$ with a $\beta^{*}=5 \mathrm{~cm}$ require further studies in order to provide a feasible design. 


\section{ACKNOWLEDGMENTS}

The authors of this paper would like to thank R. de Maria, M. Giovanozzi, J. Barranco and D. Banfi for the help provided in the use of CERN codes, A. Wolski for many useful discussions, and finally, all the valuable input from all members of the LHeC Study Group Collaboration, especially M. Klein, O. Brüning and F. Zimmermann. Work supported within the OPAC network which is funded by the European Commission under grant Agreement No. 289485.

[1] O. S. Brüning, P. Collier, P. Lebrun, S. Myers, R. Ostojic, J. Poole, and P. Proudlock, CERN Report No. CERN2004-003-V-1, 2004.

[2] ATLAS Collaboration, J. Instrum. 3, S08003 (2008).

[3] ALICE Collaboration, J. Instrum. 3, S08002 (2008).

[4] CMS Collaboration, J. Instrum. 3, S08004 (2008).

[5] LHCb Collaboration, J. Instrum. 3, S08005 (2008).

[6] http://hilumilhc.web.cern.ch/.

[7] M. Seidel, in Proceedings of 1999 Particle Accelerator Conference, New York City, USA (IEEE, New York, 1999), p. 34.

[8] S. D. Fartoukh and R. De Maria, in Proceedings of the 3rd International Particle Accelerator Conference, New Orleans, LA, 2012 (IEEE, Piscataway, NJ, 2012), p. 145.

[9] S. Fartoukh, Phys. Rev. ST Accel. Beams 16, 111002 (2013).

[10] http://madx.web.cern.ch/madx/.

[11] R. Tomás, T. Bach, R. Calaga, A. Langner, Y. I. Levinsen, E. H. Maclean, T. H. B. Persson, P. K. Skowronski, M. Strzelczyk, G. Vanbavinckhove, and R. Miyamoto, Phys. Rev. ST Accel. Beams 15, 091001 (2012).

[12] G. Robert-Demolaize, in The Advanced Optics Control workshop, CERN, Switzerland, 2015, https://indico.cern .ch/event/349643.

[13] J. L. Abelleira Fernandez et al. (LHeC Study Group), J. Phys. G 39, 075001 (2012).

[14] F. Zimmermann, in Proceedings of the International Particle Accelerator Conference, Kyoto, Japan (ICR, Kyoto, 2010), TUPEB037, p. 1605.

[15] J. Abelleira, N. Bernard, S. Russenschuck, R. Tomas, and F. Zimmermann, in Proceedings of the 2nd International
Particle Accelerator Conference, San Sebastián, Spain (EPS-AG, Spain, 2011), WEPZ013, p. 2796.

[16] R. Tomás, in The Meeting on LHeC with Daresbury group, Daresbury, UK, 2012, https://indico.cern.ch/event/207665/.

[17] M. Korostelev, E. Cruz-Alaniz, D. Newton, A. Wolski, O. Brüning, and R. Tomás, in Proceedings of the 4th International Particle Accelerator Conference, Shanghai, China, 2013 (JACoW, Shanghai, China, 2013), MOPWO063.

[18] R. De Maria, S. Fartoukh, A. Bogomyagkov, and M. Korostelev, in Proceedings of the 4th International Particle Accelerator Conference, Shanghai, China, 2013 (Ref. [18]), TUPFI014.

[19] S. Russenschuck, in The 2012 CERN-ECFA-NuPECC Workshop on the $\mathrm{LHeC}$, https://indico.cern.ch/event/ 183282.

[20] E. Cruz-Alaniz, in The EIC 2014 Workshop, http://appora .fnal.gov/pls/eic14/agenda.full.

[21] H. Wiedemann, Particle Accelerator Physics (Springer, New York, 2007), p. 802.

[22] B. Parker, in The FCC 2015 Study week, Washington, USA, 2015, http://indico.cern.ch/event/340703.

[23] S. Fartoukh and M. Giovannozzi, Nucl. Instrum. Methods Phys. Res., Sect. A 671, 10 (2012).

[24] E. McIntosh and R. de Maria, The SixDesk run environment for SixTrack, http://sixtrack-ng.web.cern.ch/ sixtrack-ng/.

[25] P. Hagen, M. Giovannozzi, J. P. Koutchouk, T. Risselada, F. Schmidt, E. Todesco, and E. Wildner, LHC Project Report No. 1123, 2008.

[26] O. Brüning, S. Fartoukh, M. Giovannozzi, and T. Risselada, LHC Project Note No. 349, 2004.

[27] R. de Maria, M. Giovannozzi, and R. Tomás, Report No. CERN-BE-2009-004.

[28] R. de Maria, S. Fartoukh, M. Giovannozzi, A. Chancé, B. Dalena, J. Payet, J. Resta-López, K. M. Hock, M. Korostelev, and A. Wolski, in Proceedings of the 4th International Particle Accelerator Conference, Shanghai, China, 2013 (Ref. [18]), WEPEA047.

[29] Y. Papaphilippou, in Proceedings of 1999 Particle Accelerator Conference, New York City, USA (IEEE, New York, 1999), p. 1554.

[30] A. Wolski, Beam Dynamics in High Energy Particle Accelerators (Imperial College Press, London, 2014), p. 385.

[31] R. Bartolini and F. Schmidt, CERN Report No. SL-Note98-017, 1998. 